force of the Cameroons Development Corporation, analysing the reasons for high labour turnover, high rates of absenteeism, low productivity, \&c. In the course of this investigation he made studies of diets and nutrition, expenditure patterns, and employment histories.

The first report of the investigation, Economic, Labour and Social Aspects of Plantations in the Cameroons, includes an Introduction by its director, Professor Richardson of Leeds University, and six sections concerned with the economic and sociological background of the labour force, nutrition and expenditure, labour problems of the Development Corporation, the supply of labour, and a study of the indigenous peoples of Victoria Division with special reference to the impact of the plantation system on the local Bakweri and other peoples in regard to agriculture, land, and social problems.

\title{
Cartes démographiques de l'Afrique
}

Sous l'égide du Comité interafricain des Sciences humaines (CCTA/CSA), s'est tenue à Bruxelles, les 5 et 6 juillet 1956, une réunion de géographes, présidée par le professeur Gourou et consacrée à l'élaboration de cartes de densité et de distribution de la population de l'Afrique au Sud du Sahara. Une quinzaine de délégués représentant les territoires belges, portugais, anglais, sud-africain et français confrontèrent leurs méthodes et leurs réalisations et adoptèrent le plan de travail dressé par le professeur Gourou.

Deux séries de cartes sont prévues: la première, au $\mathrm{I} / \mathrm{r} .000 .000$, teprésentera la distribution de la population par points, sur la base d'un point par 100 habitants. La seconde, au I $/ 2.000 .000^{\circ}$, sera tirée de la première et mettra en valeur les zones de densité par un choix de teintes appropriées.

De nombreux problèmes techniques sont ainsi posés, en particulier la collecte d'une documentation de base exacte à l'échelle administrative la plus basse (c'est-à-dire pour les territoires français, le canton) et la localisation des points de distribution dans un pays où le peuplement même sédentaire est encore instable.

L'IFAN participera activement à une telle œuvre. D'ailleurs la section de géographie, poursuivant la parution des cartes ethno-démographiques, achève la préparation de la carte $\mathrm{n}^{\circ} 2$ qui couvre le Soudan occidental, les moitiés orientales de la Guinée française et de la Sierra Leone et le Libéria.

\section{La Conférence de l'O.M.S. pour l'Afrique au sud du Sahara}

LA sixième session du Comité de l'Office Mondial de la Santé pour l'Afrique au sud du Sahara, tenu en I956 à Saint-Paul de Loanda, capitale de l'Angola, a consacré ses cinq journées de travail à l'étude du budget de l'O.M.S. et à la discussion de l'orientation à donner à l'action de l'O.M.S. en Afrique Noire.

La session, qui s'est deroulée sous la présidence du Dr Jorge Janz, professeur à l'Institut de Médecine tropicale de Lisbonne, et du Dr Dupré-Leroux, secrétaire d'État à la Santé d'Afrique du Sud, a décidé de porter en 1957 et en 1958 l'effort sur la lutte contre la lèpre et la tuberculose en Afrique. Cette campagne fait suite à celle menée en I 956 contre le paludisme et les trépanématoses. Selon les rapporteurs, la tuberculose se développe surtout dans les faubourgs ouvriers des grandes villes, et atteint deux fois plus d'hommes que de femmes. Dans les campagnes, la tuberculose semble due avant tout à la malnutrition.

C'est dans le domaine de la lutte contre la lèpre en A.É.F. que les résultats sont les meilleurs avec 120.000 cas dépistés, I I 6.000 traités, sur un total de malades évalué à 1 50.000 .

Le sixième Comité de l'Office Mondial de la Santé pour l'Afrique Noire a décidé de tenir sa prochaine réunion - en septembre 1957 - à Brazzaville et sa session de 1958 à Monrovia. 